\title{
Big Brother has evolved
}

\section{Tracking someone's movements can now be done cheaply and easily, and there are few restrictions on who can monitor whom, says Jerome E. Dobson.}

\begin{abstract}
f you're still getting used to the idea that closed-circuit television (CCTV) has turned some public spaces into a modern panopticon - the 'all-seeing' prison-like building conceived by eighteenth-century architect Samuel Bentham - then you're in for a shock. The next generation of surveillance technologies is making even George Orwell's Big Brother seem amateurish, with huge implications for
\end{abstract} privacy and personal freedom.

Although CCTV is passive

- you get spotted only if you are in front of a camera - other tools now offer constant surveillance. They are best described as human-tracking systems: devices that allow the electronic monitoring of individuals 24 hours a day, using geographic information systems (GIS), Global Positioning System (GPS) receivers and two-way communication. The technology has been around for a while. Courier companies, for example, use tracking systems to monitor goods in transit. What is new is the extent to which they are being used to monitor the movement of people, in some cases without their knowledge.

There is no shortage of available devices. A GPS receiver and radio-frequency identification (RFID) transmitter can be installed in a bracelet, tag or mobile phone, or implanted under the skin, and the carrier's coordinates transmitted to a service provider. The device's location can then be matched to any feature locatable in a GIS, such as a specific street or building. Some devices go even further, recording physiological functions such as body temperature, heart rate and perspiration.

None of this demands great technological sophistication on the user's part. Indeed, it is possible to get effective results using a standard mobile phone. Triangulating a handset's radio signals among nearby mobile-phone masts can give reasonably accurate coordinates, and some mobile phones come with GPS receivers. A laptop computer logged onto a wireless network can be tracked in the same way.

Electronic human-tracking systems have many positive applications. For example, they allow law-enforcement services to monitor offenders in their communities rather than imprison them. Families can use them to keep an eye on relatives with Alzheimer's disease in case they wander away from home. The technologies are highly effective, easy to use and relatively cheap. In fewer than five years, the annual cost of continuous surveillance of an individual has fallen from several hundred thousand dollars to less than US $\$ 500$.

This means that technology that was once exclusively the domain of national security and highstakes commerce is now available to anyone. For example, it is fairly easy for a spouse to obtain a device or service that enables him or her to follow a partner's every step; or for a parent to acquire a tracking device that can be locked to a child's wrist; or for employers to monitor the movements of their staff. Xora, a mobile-resource management company in Mountain View, California, claimed to be tracking more than 50,000 employees at 4,500 companies in 2005 (the latest year it made such information available).

Manufacturers of human-tracking systems refer to them as 'geofencing' products. Yet geofencing, if done coercively or surreptitiously, can quickly lead to 'geoslavery', in which the person doing the monitoring has significant control over the target, including the power to reprimand or punish. It is easy to see how well-intended monitoring can evolve into something troublesome. Many people would agree, for example, that parents have a right to know their children's whereabouts, but round-the-clock surveillance and control is a more dubious prospect. Similarly, every government has a right and duty to monitor suspected foreign terrorists, but tagging all immigrants would raise serious human-rights concerns.

Manufacturers cannot be held responsible for the ways in which some people might use their products, but human-tracking devices are bound to amplify some of the more extreme tendencies of human nature. How can we protect against that? A standard test should be to ask what its analogue form would have been called before GPS came along and what laws and customs applied. Was it parenting, care-giving, delivery tracking or, alternatively, incarceration, branding, stalking, slavery? These answers will help each country and culture determine how its laws, customs and institutions should be changed.

It is also crucial to address the ethical issues of human tracking in scientific research. Consider the controversy last year over a study on human mobility patterns by Marta González and her colleagues at Northeastern University in Boston, Massachusetts ${ }^{2}$. The group used information from 100,000 people's mobilephone records over 6 months to track the users without their knowledge or consent. The European telecommunications company that provided the data claims to have anonymized it. Critics, however, say anonymity does not equal consent, and geography is identity. Find where each phone spends most of the day or night and then look up the street addresses. From these it is often possible to determine the owner's name, residence and workplace. Ethical guidelines are needed to ensure that investigators understand the risks as well as the benefits of new research opportunities.

The social-networking benefits of humantracking systems will surely be substantial, for example, for friends who want to find, and stay in touch with, each other while on the move - Google Latitude offers a tracking service for free. Just as surely, the technology is bound to alter all sorts of social relationships: husband-wife, parent-child, teenager-teenager, employer-employee, government-citizen, seller-customer, researcher-subject, criminal-victim. We have entered a grand social experiment as momentous as any in our past and yet one so insidious that hardly anyone seems to have noticed. Jerome E. Dobson is in the Department of Geography at the University of Kansas, Lawrence, Kansas 66045, USA.

e-mail: dobson@ku.edu

1. Dobson, J. E. \& Fisher, P. F. Geogr. Rev. 97, 307-323 (2007)

2. González, M. C., Hidalgo, C. A. \& Barabási, A.-L. Nature 453, 779-782 (2008)

See also News Feature, page 959. 